\title{
Enhanced Knowledge Management by Synchronizing Mind Maps and Fuzzy Cognitive Maps
}

\author{
Sara D’Onofrio, Edy Portmann, Patrick Kaltenrieder, Thomas Myrach \\ Institute of Information Systems, University of Bern \\ sara.donofrio@iwi.unibe.ch, edy.portmann@iwi.unibe.ch, \\ patrick.kaltenrieder@iwi.unibe.ch, thomas.myrach@iwi.unibe.ch
}

\begin{abstract}
This paper presents a conceptual approach to enhance knowledge management by synchronizing mind maps and fuzzy cognitive maps. Using mind maps takes advantage of human creativity, while fuzzy cognitive maps can store and retrieve information expressed in natural language. Applying the concepts of cognitive computing makes it possible to gather and extract relevant information from a data pool. Therefore, this approach is intended to provide a framework that enhances knowledge management. To demonstrate the potential of this framework, a use case concerning the development of a smart city app is presented.
\end{abstract}

Keywords: Cognitive Computing, Creativity Techniques, Fuzzy Clustering, Fuzzy Cognitive Maps, Knowledge Management, Mind Maps, Smart City App, Soft Computing

\section{$1 \quad$ Introduction}

People are constantly confronted with information. By acquiring, processing and understanding information, knowledge can be created, and managing this knowledge appropriately enables us to make better decisions. At first sight, this argumentation may seem logical, even self-evident, but it contains a hidden challenge: Conducting a conversation mostly involves natural language that consists of words and sentences (i.e., jointed words). It is not very difficult to form grammatically correct sentences. However, it is rather challenging to ensure different people understand them in the same way (cf. emergent semantics [3]). Everyone's background knowledge varies [21] and, even with today's advanced information and communication technologies, it is becoming increasingly difficult to manage all the information from different sources and to take the needs of all stakeholders into account. It is becoming increasingly essential to efficiently use existing knowledge to enhance everyone's living standards, and by using connectivism (i.e., connected learning and cognition theory), people can learn from one another [20] and thus benefit from others' experiences. One way to handle this challenge and also foster this potential is to develop and build cognitive systems that help users to cope with today's ever-increasing amount of information. Cognitive computing facilitates the communication between humans and computer systems, and problem solving and 
decision-making can be improved. Thus, knowledge management (i.e., the acquisition, aggregation and representation of knowledge [19]) can be enhanced.

This paper is meant to elaborate on the framework proposed by D'Onofrio et al. [6]. It presents a more detailed insight into the idea of synchronizing mind maps (MMs) with fuzzy cognitive maps (FCMs). This framework should be able to gather and extract relevant information and thus support humans in collecting and evaluating them. This paper is an outline of a current work-in-progress, in which the authors pursue an approach relying on design science research [9]. It is a first step towards cognitive computing, according the law of parsimony. The goal is to develop cognitive systems that are able to store, connect and retrieve information like a human brain. According to Dewhurst and Conway [4], pictures are more likely to be recognized than words, which is why MMs are used to facilitate the acquisition and computation of words. Transforming MMs into FCMs enables machines to build efficient connections; therefore, retrieving the information is more efficient. The goal is a system that can think like a human and thus facilitate the communication between human and computers. Furthermore, cognitive systems should facilitate the exchange of experiences, so that people can share their knowledge (i.e., connectivism) [20].

These considerations are structured as follows: section 2 presents the theoretical background; section 3 outlines the proposed framework; section 4 illustrates this framework with a use case concerning app development; and section 5 concludes the paper.

\section{Theoretical Background}

This section explains the concepts of soft computing, creativity techniques and cognitive computing, all of which are required to understand the proposed approach.

\subsection{Soft Computing}

Soft computing is a consortium of methodologies that play an important role in the conception, design, and utilization of cognitive systems. Dividing a case into granules (i.e., clusters) [31] is a way to analyze and solve problems. By introducing fuzzy set theory [28] into a crisp clustering process, fuzzy clustering $(\mathrm{FCl})$ broadens traditional data clustering, as one element of a dataset can belong to multiple clusters. To structure and describe datasets, fuzzy logic [29] can be applied to identify similarities and differences among clusters for detecting knowledge [11] and patterns in the data [1]. FCMs can be used to represent knowledge and transfer it in a simple way, which consists of nodes (i.e., concepts) and edges (i.e., causal relationships between concepts). They model complex issues based on large amounts of data by reducing them to the essential causal dependencies. By applying fuzzy logic, FCMs can indicate how much the concepts influence one another by including edge weights within the interval $[0,1][15]$. 


\subsection{Creativity Techniques}

Creativity techniques help to collect information, look at problems from different perspectives and/or structure gathered information [8]. Based on the evaluation of Kaltenrieder et al. [12], MMs can be combined with FCMs. Their branches form a structure of interconnected nodes (similar to FCMs), allowing a structure that is hierarchical and network-oriented (see Fig. 1).

\begin{tabular}{|l|l|l|l|}
\hline Information A1 & Main Theme Bormation B1 \\
\hline \hline Information A2 & \\
\hline \hline Information C1 & Information B2 \\
\hline \hline Information C2 & Main Theme C & Information D1 \\
\hline
\end{tabular}

Fig. 1. Principle of a MM

\subsection{Cognitive Computing}

To cope with today's complex datasets [10], cognition and the principles of cognitive computing (i.e., connectivism [20], computational thinking [26] and intelligence amplification loop [13]) should be considered when constructing new systems. Cognitive computing aims to acquire, aggregate and represent data in an efficient way, so that people can manipulate and make inferences on the basis of the data [17].

\section{The Conceptual Framework}

This section addresses the proposed approach using the specified concepts above to give a better insight into the idea of the framework. Creativity techniques have been applied in various approaches in combination with FCMs (e.g., Eppler [7], Kontogianni et al. [14]), in scenario development (e.g., Stylios and Groumpos [23]) or for support in decision-making (e.g., Xirogiannies et al. [27]). Although this combination has previously been researched, there is still potential to elaborate it further. The contribution of this paper is its focus on using FCMs to process information that was explicitly acquired through MMs. As illustrated in Fig. 2 the framework consists of seven steps.

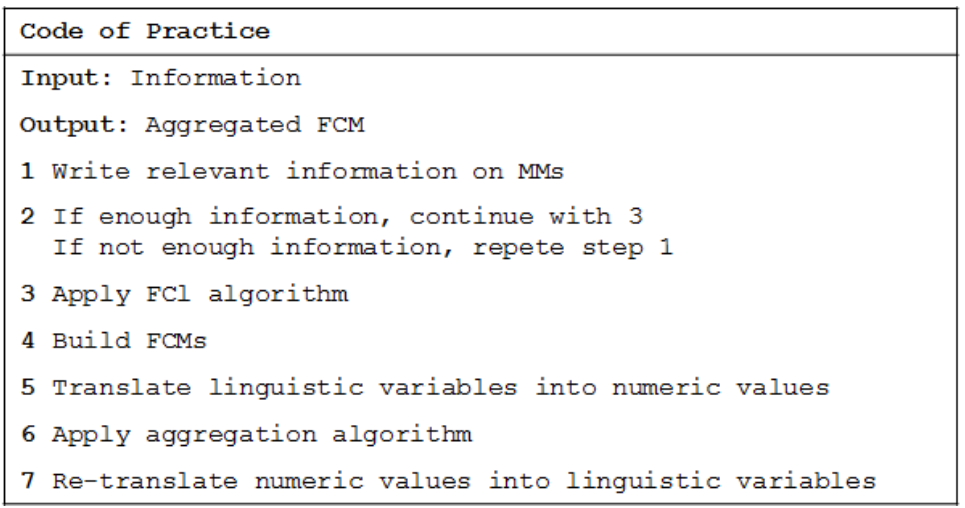

Fig. 2. Code of Practice 
The following use case is based on this approach and explains the proposed framework in more detail.

\section{$4 \quad$ Use Case: Smart City App}

The fictitious example is a tourism company that wants to develop a smart city app to optimize their business. The specification process of the relevant features is complex, as the office seeks to involve all of its stakeholders. A way to streamline this process is to apply the proposed approach (see section 3).

Step 1. Stakeholders spend time brainstorming the project "smart city app" and try to write keywords into MMs (e.g., needs, concerns, wishes). By applying fuzzy granulation, the information gathered through MMs is fractionalized into granules that support zooming-in-and-out functions (i.e., by focusing on a main theme, the underlying information appears). Several MMs can be created as a first step. An example of an MM is demonstrated in Fig. 3, showing main themes (e.g., sightseeing) and related information (e.g., guided tour).

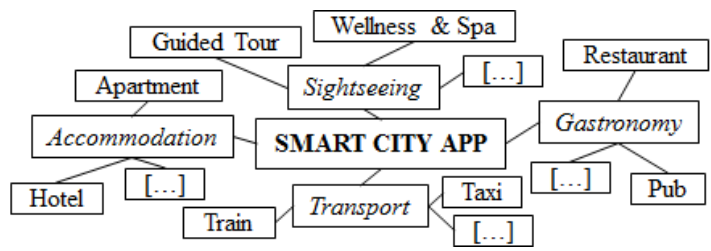

Fig. 3. Example of a MM

Step 2. When the brainstorming session is finished, the created MMs are submitted for conversion.

Step 3. Information that belongs to a certain concept with a membership degree higher than a specified level (i.e., $\alpha$-cut [25]) is extracted using the $\mathrm{FCl}$ algorithm. For this framework, a method of $\mathrm{FCl}$ can be adapted from the thesis of Portmann [18]. So, in this use case, applying fuzzy granulation [31], following clusters are automatically built: accommodation, sightseeing, transport and gastronomy (see Fig. 4 adapted from Zadeh [33]).

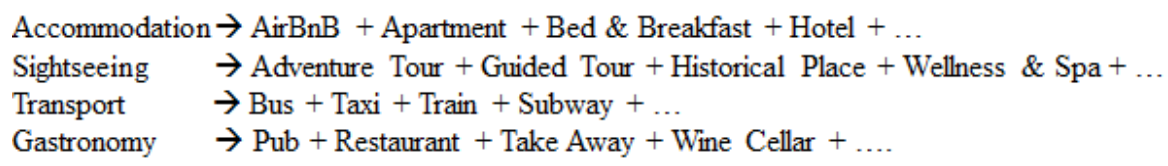

Fig. 4. Clusters

Step 4. The application of fuzzy granulation creates several main theme clusters (e.g., sightseeing) which are represented as FCMs. A possible example of extracted information from MMs to FCMs at a high level (i.e., without going into details) is depicted in Fig. 5. In this case, four main themes appear. The four axes in the square represent the fuzzy sets (i.e., main themes), whereas the points inside the square incorporate the elements of data (i.e., related information) with their membership degree to these fuzzy sets [5]. 


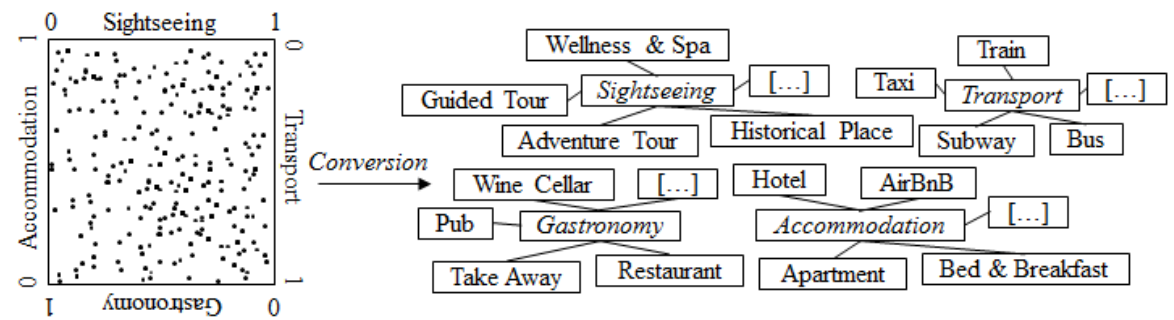

Fig. 5. Conversion

Having assigned the data elements to the fuzzy sets, the information can be converted into FCMs. An initial mathematical formulation of this process can be found in the work of Stylios and Groumpos [23].

Step 5. For the aggregation process, the linguistic variables of the created FCMs have to be translated into numeric values (e.g., with the help of computing with words (CWW) [30]), which, for now, is performed manually. Following the law of parsimony, one possibility is to use fuzzy if-then rules. Adapted from Zadeh [30], the following example can be obtained:

If Restaurant 1 is mostly booked up then Service is good.

As mostly booked up and good are imprecise descriptions containing semantic values, fuzzy sets can be used to curtail the numeric values. In this example mostly booked up can mean that approximately $80 \%$ of the time the restaurant is booked up. The term good can mean that 9 of 10 customers are satisfied with the service. Thus,

If linguistic value is mostly booked up then numerical value is $[0.8,1]$.

If linguistic value is good then numerical value is $[0.9,1]$.

The numerical values are defined based on the membership function with an interval of $[0,1]$ [29]. The more information that is available, the more connections can be built between this information, and the more accurate the fuzzy sets become; thus, better results can be obtained. Once these numerical values based on fuzzy sets have been obtained, the next step can be conducted.

Step 6. The various FCMs are aggregated into one large FCM (e.g., by taking the averages of the weights of the edges [19]) that represents all the relevant gathered information, as illustrated in Fig. 6. A mathematical formulation for this process can be found in the thesis of Stach [22]. In this case, one aggregated FCM (at a high level) with the name "smart city app" is created. 


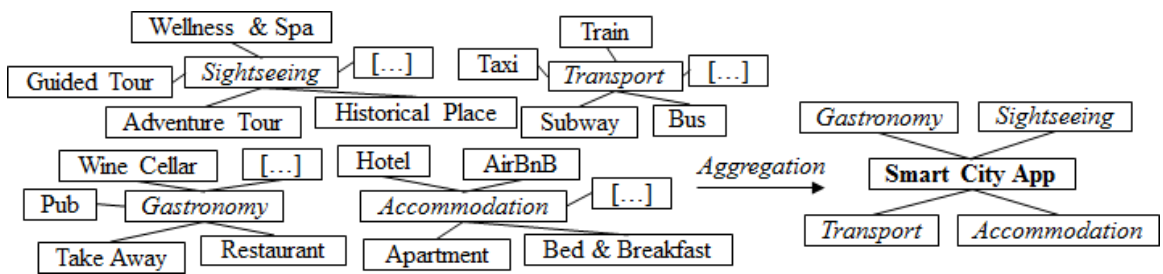

Fig. 6. From FCMs to one FCM

Step 7. The numeric values have to be re-translated into linguistic variables so humans can understand the FCM.

To summarize, the aggregated FCM consists of different levels of granularity and thus allows the users (e.g., decision-makers) to zoom in and out [19], [21]. Thus, the proposed framework is in line with the needs of the decision-makers.

\section{Conclusions and Outlook}

This paper shows an exemplary adoption of the proposed approach by gathering information, splitting it into granules, converting it to (machine-readable) FCMs and finally aggregating it into one FCM. The contribution of this paper is its focus on using FCMs to process information that was explicitly acquired through MMs and to refine the concept proposed by D'Onofrio et al. [6]. The underlying vision of this framework consists of developing and building cognitive systems that allow semi-automated reasoning. The combination of MMs and FCMs can be highly beneficial in decision-making processes, as it helps to handle complex problems by taking advantage of human creativity. Using the proposed framework may help collective intelligence [16] to arise. This framework facilitates communications between humans and computer systems and, therefore, collaborations between people. Thus, knowledge management can be improved as well.

MMs encourage people to use their creativity and express their needs [2]. Furthermore, as granulation allows the compression of data [33], MMs are appropriate to reduce the complexity of an issue to essentials keywords. MMs are only useful to a certain extent, but their combination with FCMs results in an efficient approach to complex problem situations [12]. As FCMs depict causal relationships between concepts, they are able to represent knowledge for cognitive systems in a humanlike way. Therefore, the proposed framework and the ongoing work-in-progress could provide an enhanced knowledge management system.

The authors worked with elaborated mathematical formulations of other researchers to ensure a basis for the proposed framework. These researchers have been referred in this paper. One of the next steps consists of refining these formulations in more detail and identifying algorithms that fit the presented approach.

As soon as the mathematical foundations are elaborated, the conceptual approach will be tested and evaluated. In addition, the link between MMs and FCMs should be measured. However, at this phase of the work-in-progress, it is not yet possible to make such evaluations. Nevertheless, the evaluation is an essential step in this development; thus, if this approach obtains reasonable outputs on real datasets, the 
authors will evaluate it. If the results are positive, further evaluations will be performed to find out if these outputs are better than other, simpler methods.

Furthermore, to make the aggregation of various FCMs possible, an automatic transformation from linguistic information into numeric information (and vice versa) must be developed. Concerning this process, an implementation of CWW (e.g., fuzzy if-then rules) is interesting. As words can be mathematically translated using fuzzy sets, CWW's perception of words as granules makes computation with information in natural language possible [30].

Even if MMs can gather as much information as possible from different stakeholders, this technique simply focuses on words. Therefore, other creative techniques (e.g., scenarios and user stories) are required to build meaningful sentences, as the semantic part of a sentence is more difficult to create than the syntactic part.

Furthermore, the proposed framework will be evaluated by different stakeholders to gain qualitative inputs and feedback.

\section{$6 \quad$ References}

[1] Bezdek J.C.: Pattern Recognition with Fuzzy Objective Function Algorithms. Springer Science \& Business Media, Berlin and Heidelberg (2013)

[2] Buzan T.: The Mind Map Book: Radiant Thinking - Major Evolution in Human Thought. BBC Books, London (1995)

[3] Cudré-Mauroux P.: Emergent Semantics. EPFL Press, Lausanne (2008)

[4] Dewhurst S.A., Conway M.A.: Pictures, Images, and Recollective Experience. Journal of Experimental Psychology: Learning, Memory, and Cognition, 20(5), 1088-1098 (1994)

[5] D'Onofrio S., Portmann E.: Von Fuzzy-Sets zu Computing-with-Words. InformatikSpektrum, 38, 1-7 (2015)

[6] D'Onofrio S., Zurlinden N., Portmann E., Kaltenrieder P., Myrach T.: Synchronizing Mind Maps with Fuzzy Cognitive Maps for Decision-Finding in Cognitive Cities, $9^{\text {th }}$ International Conference on Theory and Practice of Electronic Governance, Montevideo, Uruguay (2016)

[7] Eppler M.J.: A Comparison between Concept Maps, Mind Maps, Conceptual Diagrams, and Visual Metaphors as Complementary Tools for Knowledge Construction and Sharing. Information Visualization, 5(3), 202-210 (2006)

[8] Grube P.P., Schmid K.: Selecting Creativity Techniques for Innovative Requirements Engineering. $3^{\text {rd }}$ International Workshop on Multimedia and Enjoyable Requirements Engineering (2008)

[9] Hevner A.R., March S.T., Park J., Ram S.: Design Science in Information Systems Research. MIS Quarterly, 28(1), 75-105 (2004)

[10] Hurwitz J.S., Kaufman M., Bowles A.: Cognitive Computing and Big Data Analytics. John Wiley and Sons, Inc., Hoboken, New Jersey (2015)

[11] Jafar M.O.A., Sivakumar R.A.: A Comparative Study of Hard and Fuzzy Data Clustering Algorithms with Cluster Validity Indices. Proceedings of International Conference on Emerging Research in Computing, Information, Communication and Applications (2013)

[12] Kaltenrieder P., Portmann E., Binggeli N., Myrach T.: A Conceptual Model to Combine Creativity Techniques with Fuzzy Cognitive Maps for Enhanced Knowledge Management. In: Fathi M. (ed.) Integrated Systems: Innovations and Applications, pp. 131146, Springer, Berlin and Heidelberg (2015) 
[13] Kaufmann M., Portmann E., Fathi M.: A Concept of Semantics Extraction from Web Data by Induction of Fuzzy Ontologies. International Workshop on Uncertainty Reasoning for the Semantic Web (2012)

[14] Kontogianni A.D., Papageorgiou E.I., Tourkoalias C.: How Do You Perceive Environmental Change? Fuzzy Cognitive Mapping Informing Stakeholder Analysis for Environmental Policy Making and Non-Market Valuation. Applied Soft Computing Journal, 12(12), 3725-3735 (2012)

[15] Kosko B.: Fuzzy Cognitive Maps. International Journal of Man-Machine Studies, 24 (1), 65-75 (1986)

[16] Malone T.W., Laubacher R., Dellarocas C.: Harnessing Crowds: Mapping the Genome of Collective Intelligence. MIT Center for Collective Intelligence (2009)

[17] Modha D.S., Ananthanarayanan R., Esser S.K., Ndirango A., Sherbondy A.J., Singh R.: Cognitive computing. Communications of the ACM 54(8):62-71 (2011)

[18] Portmann E.: The FORA Framework: A Fuzzy Grassroots Ontology for Online Reputation Management. Springer, Berlin and Heidelberg (2013)

[19] Portmann E., Pedrycz W.: Fuzzy Web Knowledge Aggregation, Representation, and Reasoning for Online Privacy and Reputation Management. Fuzzy Cognitive Maps for Applied Sciences and Engineering. Springer, Berlin and Heidelberg (2014)

[20] Siemens G.: Connectivism: A Learning Theory for the Digital Age. International Journal of Instructional Technology and Distance Learning, 2(1), 3-10 (2005)

[21] Sowa J.F.: Knowledge Representation - Logical, Philosophical, and Computational Foundations. Course Technology, Cengage Learning (2000)

[22] Stach W.: Learning and Aggregation of Fuzzy Cognitive Maps - an Evolutionary approach. Ph.D. Thesis, University of Alberta (2010)

[23] Stylios C.D., Groumpos P.P.: Mathematical Formulation of Fuzzy Cognitive Maps. Proceedings of the 7th Mediterranean Conference on Control and Automation (MED99) Haifa, Israel (1999)

[24] Van Vliet M., Kok K., Veldkamp T.: Linking Stakeholders and Modellers in Scenario Studies: The Use of Fuzzy Cognitive Maps as a Communication and Learning Tool. Futures, 42(1), 1-14 (2010)

[25] Werro N.: Fuzzy Classification of Online Customers. In: Meier A., Pedrycz W., Portmann E. (eds.) Fuzzy Management Methods. Springer, Berlin and Heidelberg (2015)

[26] Wing J.M.: Computational Thinking. Communications of the ACM, 49(3), 33-35 (2006)

[27] Xirogiannis G., Stefanou J., Glykas M.: A Fuzzy Cognitive Map Approach to Support Urban Design. Expert Systems with Applications, 26(2), 257-268 (2004)

[28] Zadeh L.A.: Fuzzy Sets. Information and Control, 8, 338-353 (1965)

[29] Zadeh, L.A.: Fuzzy Logic. IEEE Computer, 21(4), 83-93 (1988)

[30] Zadeh L.A.: Fuzzy Logic $=$ Computing with Words. IEEE Transactions on Fuzzy Systems, 4(2), 103-111 (1996)

[31] Zadeh L.A.: Toward a Theory of Fuzzy Information Granulation and Its Centrality in Human Reasoning and Fuzzy Logic. Fuzzy Sets and Systems, 90, 111-127 (1997)

[32] Zadeh L.A.: Some Reflections on Soft Computing, Granular Computing and Their Roles in the Conception, Design and Utilization of Information/Intelligent Systems. Soft Computing, 2, 23-25 (1998)

[33] Zadeh L.A.: From Computing with Numbers to Computing with Words - from Manipulation of Measurements to Manipulation of Perceptions. Annals New York Academy of Sciences, 929(1), 221-252 (2001) 